\title{
Monitor UP: New Solution for Grid Frequency and Voltage Functional Monitoring
}

\author{
Marcello Testori ${ }^{1}$, Olivier Lamquet ${ }^{1}$, David Dresco ${ }^{1}$, Adriano Gubernali ${ }^{2}$
}

\begin{abstract}
Transmission System Operators (TSOs) are in charge of the security, safety and reliability of the power system. One aspect of this responsibility is to verify that all the grid connected units are compliant with the Grid Code. Within all the technical rules described in the Grid Code, ancillary services for voltage and frequency controls have a relevant part since they have a direct impact on power system reliability.

The Italian TSO Terna has initiated a project with the purpose of monitoring the performances of the frequency and voltage controls provided by the main power units connected to the Italian grid. CESI has been asked to assist Terna in the definition and implementation of this project. CESI has developed the MONITOR UP application which implements Key Performance Indicators (KPIs) for the ancillary services and provide grid operators with information about the grid overall control of voltage and frequency.

The analyses performed by the application are both static and dynamic. The elaborations are based on measurements coming from Terna national control system. Based on its input, Monitor UP computes KPIs which can characterize the performances of the frequency and voltage controls supplied by the power units. The scope of this article is to illustrate the analyses of the measurements, theoretical studies and software
\end{abstract}

\footnotetext{
${ }^{1}$ CESI - Via R. Rubattino, 54 - 20134 Milan, ITALY (e-mail: marcello.testori@cesi.it, olivier.lamquet@cesi.it, david.dresco@cesi.it)

${ }^{2}$ Terna S.p.A. - Rete Elettrica Nazionale Roma - Viale Egidio Galbani, 70 -00156 (e-mail: adriano.gubernali@terna.it)
}

realizations of Monitor UP application.

\section{Keywords}

Power system, grid, frequency control, voltage control, monitoring, Grid Code, ancillary services, Key Performance Indicator, statistical and real-time analysis, measurements.

\section{Introduction}

Terna, as Italian transmission system operator, is in charge of the safe, secure and reliable operation of the national transmission and sub-transmission grid (380, 220 and $150 \mathrm{kV}$ ), which represent about $300 \mathrm{OHL}, 3$ submarines cables and 9 cross borders tie lines continuously monitored for a total of about $63.500 \mathrm{kms}$. A relevant task to achieve this goal is to assess the compliance of generating units with the requirements under the national Grid Code [2]. As stated by [3], this activity should be performed not only during units connection phase but periodically throughout the all lifetime of the units, especially after any failure, modification or replacement of equipment that may have impact on units' compliance. Within the requirements, ancillary services and especially voltage and frequency controls have a relevant role for power system reliability.

One of the main aspects to successfully achieve such reliability is to have part of these services delivered automatically and autonomously (i.e. without any coordination) by a great number of qualified units: within the control systems of these units (governor, exciter, ... ), dedicated functions implement part of these ancillary services (e.g. frequency droop, AVR,...). The drawbacks of such methodology are the large territorial dispersion of the services' provision and therefore the difficulties in taking

This is an Open Access article distributed in accordance with the Creative Commons Attribution Non Commercial (CC-BY-NC-ND 4.0) license, which permits others to copy or share the article, provided original work is properly cited and that this is not done for commercial purposes. Users may not remix, transform, or build upon the material and may not distribute the modified material (http://creativecommons.org/licenses/by-nc/4.0/) 
under control the actual and proper delivery of these services by each qualified unit.

Two methods are usually used to verify the compliance of the delivered services with the grid code requirements:

- $\quad$ Auto certification: a power unit owner is responsible for ensuring unit compliance with grid code requirements throughout the lifetime of the facility. In addition, the Italian grid code asks units owners to periodically (3-years process) control and certificate that their units are compliant with the grid code requirements, at least for the main relevant services related to power system operation during emergencies. This auto-certification shall be based on the results of dedicated tests performed autonomously or assigned to third-parties. The tests procedures to follow are publicly available as annexes of the grid code itself.

- $\quad$ Compliance testing: the Italian grid code entitles Terna to request units' owners to carry out compliance tests. The tests are usually performed according to the same public tests procedures as used for auto-certification, but additional tests can be required for in-depth analysis of specific technical issues related to compliance. Compliance testing is usually required by Terna to grant an operational notification to a new power unit, but can also be required when a unit abnormal behaviour is put in evidence by Terna dayby-day network operation analysis or during the postanalysis of large grid transients.

Monitor application has been designed to represent a platform which continuously monitors the performance of ancillary services provided by those generating units which are qualified for voltage and frequency controls. Monitor also provides operators with some KPIs related to the quality of services provided by these units. The final scope is to early identify malfunctioning units through their KPIs going beyond warning thresholds, and consequently require compliance testing for investigation and before these malfunctions can potentially impact on power system reliability, especially during emergency transients.

\section{Monitoring application and realization}

Monitor UP's scope is to improve the electrical system reliability, helping the TSO to early identify any possible issue on the controlled grid. Monitor UP application is currently used as a support for network analysis department but it has been designed with the possibility of a future integration within the NCC (National Control Center) [1]. Furthermore the application provides a statistical analysis based on historical data collected at Power Plant level. Since data are topologically not centralized on the Italian territory the regulations are locally implemented on the generators governors - the application has been developed to concentrate all data that must be recorded and analyzed. The application builds a mySQL database that can be filled with data provided by TERNA via a secure FTP connection or directly linked to the TSO real-time databases. The local database stack format is realized through Database Management System with the ODBC standard connector drivers. The whole software required by the application is compatible with Windows Operating System and it is implemented on the latest National Instruments LabVIEW release.

\subsection{Principal application execution}

Since Monitor UP is evolving with TSO's needs, the application is developed with the purpose of being flexible at any possible modification. For this reason there are different asynchronous tasks running independently which are linked to the local database. Through it the tasks communicate one with another and make possible the analyses (III. , IV. ).

Principally there are three tasks which are continuously running when the application starts. The first one is the DB loader which has the purpose of filling the local Database with the data provided by the Remote Terminal Units of TSO system. The second is the UP Calculation task which performs all the elaborations required for the statistical and real time analysis (III. , IV. ). The results of this elaboration are stored in the local database.

The third asynchronous task is the Man Machine Interface which provides a grid synoptic with the main relevant measures and results, alarms, diagnostic functions, configuration parameters and transients' visualization. The input data of the main page are taken from the database filled by the aforementioned two tasks.

Figure 1 shows Monitor UP application's main page.

\subsection{Subordinate application execution}

LabVIEW programming language supports the integration with a large variety of programs and applications of Windows operating systems. Thus in the application different programs support the user to understand grid regulation issues or to report the data analyses (III. IV. ).

Monitor UP implementation offers the integration with Microsoft Office applications. Through Monitor's Run-Time Menu, it is possible to output the KPI analysis (III. B, III. D) in MS Word format (with relative graphs 


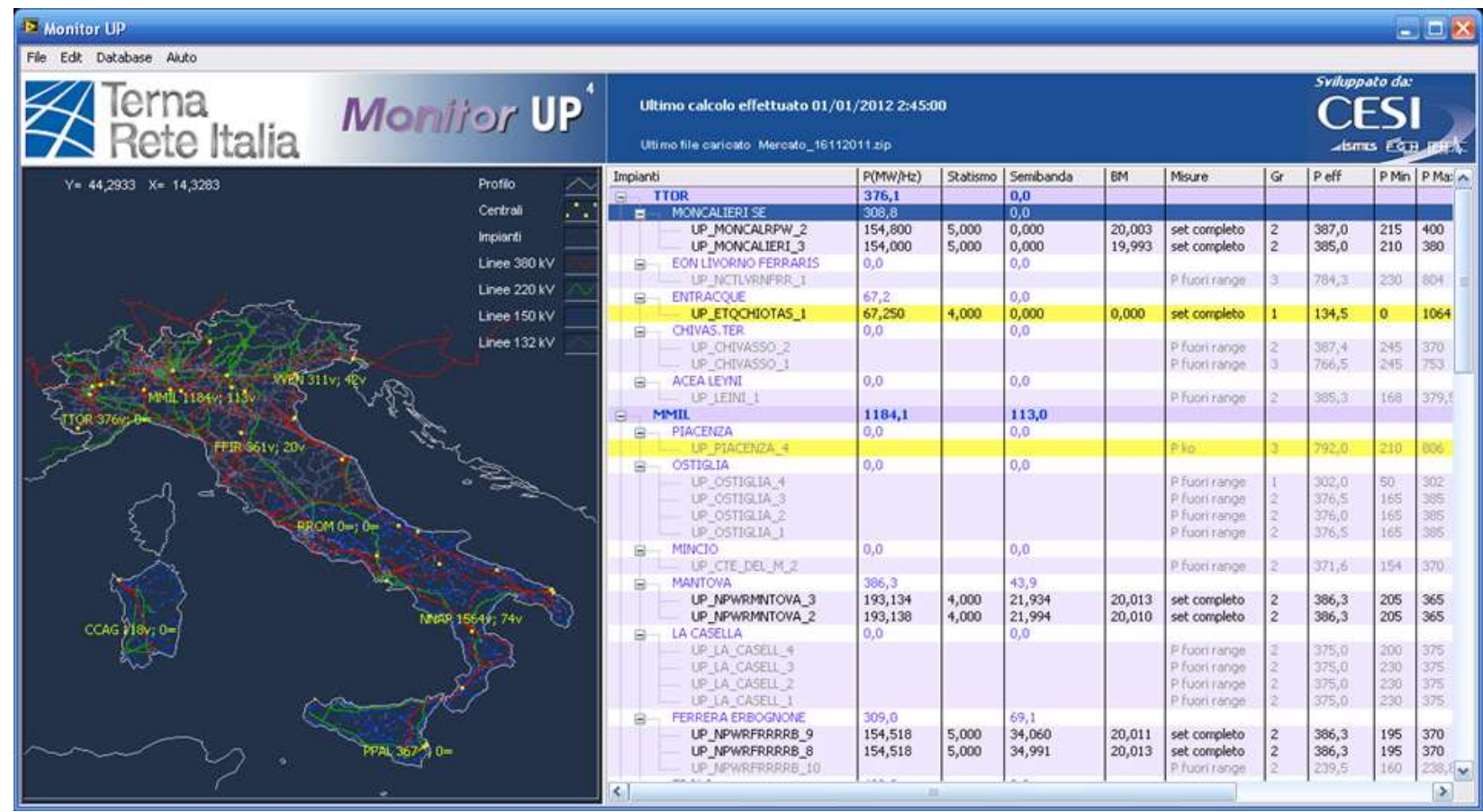

and charts) and exporting all the records in commaseparated values or text formats.

Another relevant feature is the Google Services integration (Fig. 2). It allows the user to launch Google

\section{Frequency monitoring}

Frequency ancillary services' efficiency is strongly dependent on the characteristics of power generator and power control systems of the generating units. Furthermore, the actual performances of power systems can be different from design because of process and ageing reasons or because of wrong control settings. To understand the causes of these possible non-compliances, Monitor UP application performs both static and dynamic analysis calulating Key Performance Indicators to help online and offline investigations.

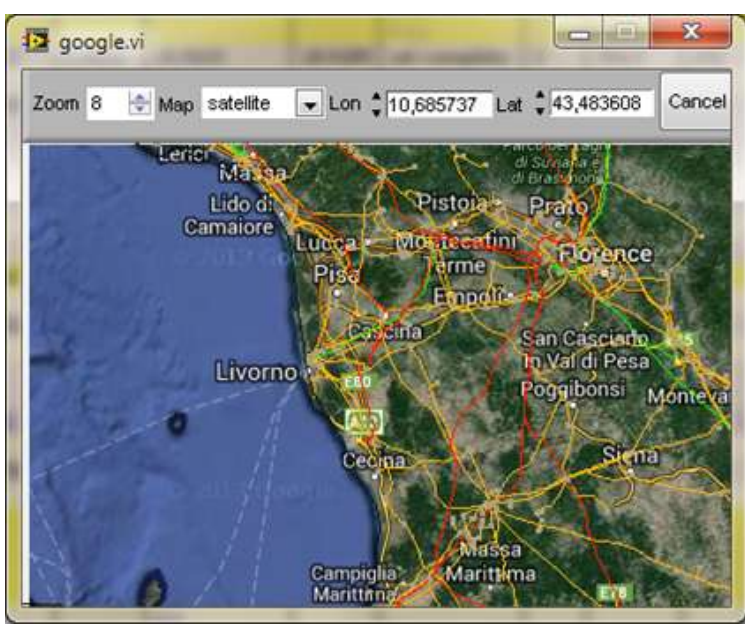

Fig. 2. Google Maps feature
Maps through a Web interface which provides interactive maps, satellite/aerial imagery of the Italian country.

Fig. 1. Monitor UP application's main page

\subsection{Mathematical approach and static analysis}

The whole theoretical mathematical approach considers each generating unit as one MIMO (Multiple Inputs, Multiple Outputs) system. The input vector $\left(u_{n}\right)$, state vector $\left(x_{n}\right)$ and output vector $\left(z_{n}\right)$ of the model are respectively:

$$
\begin{gathered}
u_{n}=\left[\begin{array}{c}
P_{0, n} \\
\Delta f_{n} \\
L_{n} \\
S B_{n}
\end{array}\right] \\
x_{n}=\left[\begin{array}{c}
P_{a u x, n} \\
C_{R S} \\
C_{R P} \\
C_{B M} \\
P_{e, n}
\end{array}\right]+w_{n} \\
z_{n}=\left[P_{n}\right]+v_{n}
\end{gathered}
$$

Where: 
- $n$ : sample of the real-time clock

- $\quad P_{0, n}$ : unit load reference at the n-th sample

- $\Delta f_{n}$ : grid frequency deviation at the $\mathrm{n}$-th sample

- $L_{n}$ : Terna remote control signal for frequency secondary control at the $\mathrm{n}$-th sample

- $S B_{n}$ : unit half band of secondary frequency control reserve at the $\mathrm{n}$-th sample

- $\quad P_{a u x, n}$ : electric power of the auxiliary services of the unit at the n-th sample

- $C_{R S}$ : unit corrective coefficient on secondary frequency control contribution

- $C_{R P}$ : unit corrective coefficient of primary frequency control contribution

- $C_{B M}$ : corrective coefficient on unit dead band on frequency control

- $\quad P_{e, n}$ : unit active power at the n-th sample

- $\left[P_{n}\right]$ : best estimate of unit active power at the $\mathrm{n}$ th sample

- $w_{n}$ : white Gaussian noise component representing the uncertainty of the model at the n-th sample

- $\quad v_{n}$ : white Gaussian noise component representing the error of the measurements at the n-th sample

At low frequency stimulations (static analysis) inputs, states and outputs are linked by the following expression:

$$
P_{e}=P_{0}+P_{a u x}+C_{R P} R P+C_{R S} R S
$$

Where unit primary $(R P)$ and secondary $(R S)$ frequency control contribution assume the following expression:

$$
\begin{aligned}
& R P=k_{n}\left(\Delta f-\left(1-k_{R B M}\right) C_{B M} B M\right) \\
& R S=L S B
\end{aligned}
$$

Where:

- $k_{n}$ : nominal unit regulation energy

- $\quad k_{R B M}$ : dead band recovery index (0 or 1$)$

- $\quad B M$ : death band value

The scope of the analysis is to estimate the state vector $\mathrm{xn}$ of the MIMO system by means of the Extended Kalman state Observer (EKO).

The problem to solve is to adapt continuously the states of the model according to measurements. The problem has been addressed from an estimator of the nonlinear state which also provides the possibility of estimating, in addition to the states, even the above mentioned parameters. The parameters actually have variations due to the operating point and the system must compensate errors due to the model linearization adopted.

The application uses techniques of observation / filtering to perform the estimation of the state variables based on measurements from the generating units provided by the TSO, and to identify the corrective parameters, thus adapting the model to different operating conditions.

For these purposes it is used the Extended Kalman Observer (EKO), considering the correction parameters as an extension of the state of the system to which it is associated no dynamic. According to Kalman theory [5] [6], Monitor UP performs the linearization on the aposteriori estimation at the (n-1)-th sample for the state equation and the a-priori estimation at the $\mathrm{n}$-th sample for the output equation. Consequently the system took in consideration becomes linear:

$$
\begin{gathered}
x_{n}=\hat{x}_{n-1}+\left[\begin{array}{ccccc}
1 & 0 & 0 & 0 & 0 \\
0 & 1 & 0 & 0 & 0 \\
0 & 0 & 1 & 0 & 0 \\
0 & 0 & 0 & 1 & 0 \\
\frac{\partial P_{e}}{\partial P_{a u x}} & \frac{\partial P_{e}}{\partial C_{R S}} & \frac{\partial P_{e}}{\partial C_{R P}} & \frac{\partial P_{e}}{\partial C_{B M}} & 0
\end{array}\right]\left(x_{n-1}-\hat{x}_{n-1}\right)+\varepsilon_{\mathrm{n}-1} \\
z_{n}=\bar{z}_{n}+\left[\begin{array}{lllll}
0 & 0 & 0 & 0 & 1
\end{array}\right]\left(x_{n}-\bar{x}_{n}\right)+\eta_{\mathrm{n}}
\end{gathered}
$$

Where:

- $\hat{x}_{n-1}$ is the a-posteriori estimation

- $\bar{z}_{n}$ is the a-priori estimation

- $\varepsilon_{\mathrm{n}-1}$ new casual independent variable with zero mean value

- $\quad \eta_{\mathrm{n}}$ new casual independent variable with zero mean value

And

$$
\left\{\begin{array}{l}
\frac{\partial P_{e}}{\partial P_{a u x}}=1 \\
\frac{\partial P_{e}}{\partial C_{R S}}=R S \\
\frac{\partial P_{e}}{\partial C_{R P}}=R P \\
\frac{\partial P_{e}}{\partial C_{B M}}=\operatorname{sign}(\Delta f) C_{R P} k_{n}\left(1-k_{R B M}\right) B M \text { per }|\Delta f|>C_{B M} B M
\end{array}\right.
$$

The EKO directly outputs the Key Performance Indexes related to the static performances of the system analyzed. 


\subsection{KPI static analysis definition}

With respect to the technical rules defined in [2] and [4], the application performs the calculation of the following indexes:

- Frequency Containment Reserve Droop Index (FCRDI) is an indicator of the static frequency droop characteristic of a generating unit and corresponds to the $C_{R P}$ coefficient described in $\$ 3$. A value lower than 1 p.u. (or 100\%) indicates a generating unit whose contribution to primary frequency control is lower than the expected value, thus put in evidence an underperforming unit.

- Frequency Containment Reserve Dead-Band Index (FCRDBI) is an indicator of the intentional dead band setting on the primary frequency regulation. FCRDBI corresponds to the $\mathrm{C}_{\mathrm{BM}}$ coefficient defined in $\S 3$. A value lower than 1 p.u. (or $100 \%$ ) indicates a dead band lower than the expected value, thus put in evidence an over-performing unit.

- Frequency Recovery Reserve Index (FRRI) is an indicator of the secondary frequency control reserve actually provided by the generating unit output. This value should be compared to the value agreed with the TSO. FRRI corresponds to the $\mathrm{C}_{\mathrm{RS}}$ coefficient defined in $\$ 3$. A value lower than $100 \%$ indicates a unit secondary frequency control reserve lower than the expected value, thus put in evidence an underperforming unit.

\subsection{Dynamic analysis}

The Italian and European Grid Code [2][4] define the timing for the delivery of the primary control contribution due to the frequency deviation:

- $\quad$ Half of the whole contribution must be delivered within 15 seconds from the frequency variation,

- $\quad$ The whole contribution must be delivered within 30 seconds from the frequency variation.

This definition of the performance identifies the step response as a method for the characterization of the dynamic behaviour of the generating units during frequency transients.

According to this point, Monitor UP application records every grid transient and, basing on these recorded transients, performs the real-time analysis of the dynamic behavior of every generating unit. Transient recording are triggered by instantaneous frequency variation going beyond settable thresholds. The analysis is based on the ratio between the actual power contribution (FCR) and the estimated one (EFCR) 15s and 30s after the trigger. FCR and EFCR are calculated as follows:

$$
\begin{aligned}
& F C R_{n}=P_{e, n}-P_{0, n}-P_{a u x, n}-C_{R S} R S_{n} \\
& E F C R_{n}=C_{R P} k_{n}\left(\Delta f_{n}-\left(1-k_{R B M}\right) C_{B M} B M\right)
\end{aligned}
$$

It is worth to note that the analysis is made at n-th sample. The application estimates then the following coefficient C:

$$
C=\frac{1}{2}\left(\frac{F C R_{n \mid 15 s}}{E F C R_{n \mid 15 s}}+\frac{F C R_{n \mid 30 s}}{E F C R_{n \mid 30 s}}\right)
$$

\subsection{KPI dynamic analysis definition}

The Frequency Containment Reserve Activation Time Index (FCRATI) index is an indicator of unit dynamic contribution to primary frequency control. It corresponds to a parametric weighted average of the coefficient $\mathrm{C}$ defined in $\$ 3.3$. A value lower than $100 \%$ for this index indicates a slow dynamic behaviour with respect to expected dynamics, thus put in evidence an underperforming unit

\section{Voltage Monitoring}

Monitor UP provides also information about the voltage behaviour of generating units. The voltage control analyses performed by Monitor are quite different from those regarding the frequency control. The scope of the analyses is to offer to operators an intuitive visualization of all the measurements involved in voltage control within the declared capabilities of active and reactive power, machine terminals' voltage, High bus bar Voltage.

From a dedicated HMI, the user can choose the generator connected to the Italian grid and the time frame for which the analysis will be provided. There is not a calculation of KPI factors such as those of frequency regulation; in this way Monitor acts as a data collector that allows a simple and effective visual representation of the generator performances for what concerns the voltage regulation. The final scope is to provide operational data in order to control if generating units reactive power capabilities are fully exploited or if there is margin for optimization.

With the increasing growth of RES and DER generation, there is an increasing need of inductive reactive power at transmission level to compensate the capacitive reactive power produced by OHL. On the transmission grid, most of the generation is bulk generation which is grid connected through synchronous generators and the reactive power range is usually narrower in under-excitation than in over-excitation; 
therefore the full use of generators capabilities is therefore becoming an important element to keep voltage within its normal operating range. The following figure gives an overview of the main available data representation.

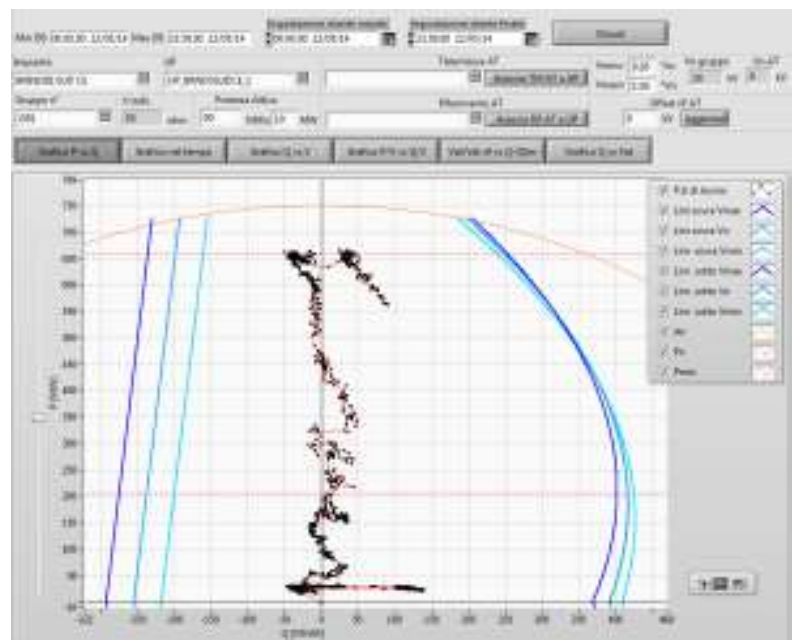

Fig. 3. Voltage regulation main page with $P / Q$ chart

The previous representation plots the operating points on a P/Q chart. On this chart the user can compare the operating points to the capability curves (blue lines) that the plant's owner has declared to TERNA.

The capability curves are $2^{\text {nd }}$ degree expressions and define the limitations on the P/Q chart:

$$
Q_{L I M \pm}=f\left(P, V, P^{2}, V^{2}\right)
$$

This representation is useful for time frame in which the grid voltage condition requires the generator to supply reactive power (in over- or under-excitation).

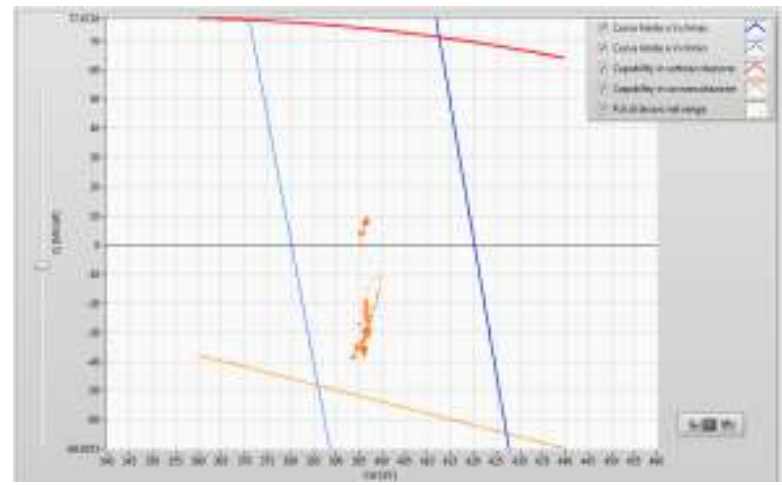

Fig. 4. Q/HighVoltage Chart
The previous chart has the reactive power on $\mathrm{Y}$-axis and the $\mathrm{HV}$ bus bar voltage on $\mathrm{X}$-axis. Beside the operating points, it plots the limitation curves related to capability (blue lines) and minimum/maximum machine terminal voltage (red/orange lines).

With the analysis showed in Figure 3 and Figure 4 (and all the others provided by the tool) it is possible to check whether the machine uses all the theoretically reactive regulation capacity.

\section{Conclusion and future developments}

The present article pointed out the importance of a real time application for monitoring the behaviour of power plants. Even if the current tool uses SCADA remote measures (sampling time $4 \mathrm{sec}$ ), in the future the tool will be supplied by PMU (Phase Measurement Unit) apparatus sited in the substations and in the power plants (sampling time $100 \mathrm{~ms}$ ). As a consequence the accuracy of calculated KPIs will be improved. In parallel way, in the future the total primary and secondary reserve calculated in real time by the tool will be used by NCC operators to make decision on activation of additional active power reserve in order to prevent big unbalances (that is big Area Control Error, ACE).

\section{References}

[1] T. M. Papazoglou, T. Babnik, U. Bezerra, C. Cattaneo, S. Gasperic, J. Kostevc, R. Mihalic, U. Rudez, G. Taranto, M. Testori, J. Vieira, "On Voltage and VAr Support in Electric Power System Operation", February 2014, No. 272 ELECTRA

[2] "Code for transmission, dispatching, development and security of the grid" as set forth in Article 1, paragraph 4 of Prime Ministerial Decree 11 May $2004-11 / 05 / 2004$.

[3] "ENTSO-E Network Code for Requirements for Grid Connection Applicable to all Generators", www.entsoe.org, 16/10/2013

[4] "Network Code on Load-Frequency Control and Reserves", www.entsoe.eu, 28/06/2013.

[5] R. E. Kalman "A New Approach to Linear Filtering and Prediction Problems", Transaction of the ASME Journal of Basic Engineering, pp. 33 - 45, 1960.

[6] G. Welch e G. Bishop "An introduction to the Kalman Filter”, SIGGRAPH 2001, 2001 